\title{
Validating LES for Jet Aeroacoustics
}

\author{
James Bridges* \\ NASA Glenn Research Center, Cleveland, $\mathrm{OH}$
}

\begin{abstract}
I. Abstract
Engineers charged with making jet aircraft quieter have long dreamed of being able to see exactly how turbulent eddies produce sound-and this dream is now coming true with the advent of large eddy simulation (LES). Two obvious challenges remain: validating the LES codes at the resolution required to see the fluid-acoustic coupling, and the interpretation of the massive datasets that result in having dreams come true. This paper primarily addresses the former, the use of advanced experimental techniques such as particle image velocimetry (PIV) and Raman and Rayleigh scattering, to validate the computer codes and procedures used to create LES solutions. It also addresses the latter problem in discussing what are relevant measures critical for aeroacoustics that should be used in validating LES codes. These new diagnostic techniques deliver measurements and flow statistics of increasing sophistication and capability, but what of their accuracy? And what are the measures to be used in validation? This paper argues that the issue of accuracy be addressed by cross-facility and cross-disciplinary examination of modern datasets along with increased reporting of internal quality checks in PIV analysis. Further, it is argued that the appropriate validation metrics for aeroacoustic applications are increasingly complicated statistics that have been shown in aeroacoustic theory to be critical to flowgenerated sound.
\end{abstract}

\section{Introduction}

\section{A. The Problem}

Typical aeroacoustic problems are bedeviled at several levels. First, the fluid flow generating the acoustic field is turbulent, and the description of this flow has a high degree of freedom. That is to say in classical engineering terms that the flow and its associated sound is described by a large number of modes over many scales. A subset of aeroacoustic problems, typically involving resonance, can be described more simply, but from an engineering perspective if the flow is simple then the method of manipulating the flow to enhance or remove the sound is also simple. Second, the sound is the result of slight phase imperfections from the compressibility of the flow. Indeed, if the flow were truly incompressible there would be no noise as all dilatations would cancel out. Third, and most significant for engineers attempting to predict the acoustic field, the sound is such a small part of the solution that it is easily lost to approximations used in analysis. Thus, the field of aeroacoustics is still a research topic instead of being an engineering science.

Of the approaches used on aeroacoustics all run into the problems mentioned above. Empirical approaches are largely successful for cases where the turbulence has simple scaling with the geometry such as simple jets and turbulent boundary layer noise. However, make a change to the geometry that changes the turbulence beyond the simple description underlying the noise model and the empirical noise model falls apart. Think modifications to nozzle geometries or fuselage-wing interfaces. Most theoretical approaches have problems handling the multitude of scales and ranges of magnitudes, making it difficult to avoid throwing the baby out with the bathwater in approximations. Acoustic analogies have been as successful as any theoretical approach, but underlying the models developed from these approaches are assumptions about the connection between the space-time correlations of turbulent quantities and the acoustic field. This is the part where a fraction, roughly one in a million in a jet, of the turbulent kinetic energy is converted to acoustic energy. And this coupling is dependent on the mean flow and observer location that is usually handled using Green's functions. The simplifications required to produce a valid Green's function further limit the usefulness of this approach for a general engineering tool, and the modeling of

\footnotetext{
* Acoustics Branch, NASA Glenn Research Center, Associate Fellow AIAA.
} 
turbulent space-time correlations may hide other descriptions of the turbulent flow-acoustic generation process that could lead to new noise reduction concepts.

\section{B. A Potential Answer}

The application of LES holds great promise for aeroacoustics, from improving fundamental understanding of noise generation, to improvements in source modeling for acoustic analogies, to practical prediction and design of exhaust systems. When done properly, LES should be able to simulate the complexity of the flow with a large enough range of motion and with enough accuracy to track the physics embodied in the Navier-Stokes equations that captures the transfer of energy from turbulent to acoustic modes. It appears that even complicated geometries and wide ranges of compressibility are possible, if not now then soon. When LES is done properly, researchers seeking a better way to describe how fluid fluctuations become sound will be able to explore all manner of descriptors of the flow, all manner of statistics. When LES is done properly, engineers wanting to predict the noise field of a machine with fluid flow will get reliable answers. When LES is done properly, optimization tools that manipulate the turbulent eddies themselves can be employed to look for near-omniscient noise reduction strategies. All this, when LES is done properly.

\section{The Problem with the Answer}

However, before any of these promises can be realized the LES technique itself must be validated to understand its limitations. What is meant by 'done properly' is not only whether the solutions very close to reality, but whether the discrepancy from reality is known and the accuracy of the answers derived from the simulation is known. For simple statistics, this is called uncertainty analysis, and is quantifiable. In the more complex solutions and in the insights obtained from such solutions this rigor may not be possible for some time. Perhaps the best that can be achieved is a layered approach to validation, starting with the simplest statistics and progressing to the more complex and acoustically relevant. This, plus internal, perhaps ad-hoc, measures of accuracy provided by the LES code, may be what is possible to establish how much the answers and insights from LES can be trusted.

Beyond the problem of validation is the problem of interpretation. Anyone who has worked with LES understands the difficulty in managing such large datasets. Today, data storage limitations often force the numericist to pre-select what parts of the flow will be saved for analysis, at least in time. Even having all the raw flow data, manipulating the data to show some measure or quantity is both the promise and the curse of LES. Promise, because it frees researchers to creatively propose new ways of looking at the problem; curse, because each manipulation creates an entity whose validity is unknown. Again, perhaps the best approach is to validate the LES simulations to enough detail that most misleading observations will be caught by some validated measure.

\section{What to measure?}

Given the success of empirical scaling laws for aeroacoustic problems, successful LES codes must be able to accurately compute the flow so that basic measures of mean velocity, temperature, and density are valid. The robustness with which the noise from a round jet can be predicted just from its ideally expanded velocity requires as much. Beyond this we look to acoustic analogy theory to sort out what are important quantities governing the production of sound from turbulent flow. It is important to review briefly the basis for acoustic analogies to understand how these quantities arise.

In a nutshell, all acoustic analogies are attempts to transform the Navier-Stokes equations into the form of a wave equation with whatever is not a wave-like operator placed on the right-hand side; these are classically considered the 'source'. The reason there are so many acoustic analogies is that so many transformations are possible. The tools of classical acoustics are then brought to bear to produce a formal solution to the wave equation. Given the complexity of the 'source' term this solution is rarely very insightful. The next step in trying to use the analogy to obtain a useful solution is to start sorting out the terms on the right-hand side, keeping those which are dominant and making approximations for them so that the numerical machinery can be worked through the formal solution to obtain an actual prediction of the sound field. In principle, the quantities on the right-hand side of the analogy are mathematically those things that give rise to acoustic waves, and are a good place to look for important measures for validating LES solutions.

From this point the discussion will be restricted to the aeroacoustic problem of jet noise, although much of it may apply to the problem of sound generated by turbulence over solid surfaces as well. Over the course of decades of theoretical development some acoustic analogies have been found to be more useful than others in constructing predictive tools. Goldstein \& Leib [1] and Khavaran [2], have shown that fluctuations of velocity are the leading terms in cold jets that need to be modeled to predict far-field sound autocorrelation, arguably the final statistic of interest in this context. When the jet is heated, velocity fluctuations begin to lose dominance and turbulent enthalpy 
fluctuations and their correlations with the velocity become more important. In particular, the actual quantities that arise in the formal solution for far-field sound spectra are the space-time correlations of Reynolds stresses, enthalpy, and enthalpy-velocity. Thus, these quantities are the ones that experimentalists should strive to measure and LES numericists should match after the basic mean and variance statistics of the LES simulation have been validated.

Several layers of validation have been, and continue to be, pursued in the aeroacoustics community. NASA researchers are contributing through advanced diagnostics of turbulent velocity, temperature, and acoustics. The next section will review many of these developments. We have acquired and established uncertainties for simple velocity statistics, namely mean and variance, in a wide range of jet flows, primarily for round jets, using particle image velocimetry (PIV). Recently, experiments to measure turbulent temperature statistics, including correlations of velocity-temperature and space-time correlations of temperature have been performed, adding to the database available for validation. In all cases, acoustic far-field spectra have been acquired to complete the flow-acoustic validation suite. Extending the parametric space into geometric variations, a similar, though more limited, set of data has been established for simple chevron nozzles with an eye to using them for CFD (and now LES) validation.

With the support of NASA's Aeronautics Mission Directorate, researchers at NASA's Glenn Research Center have gone from measurements of mean and variance of velocity to measures of spatial and space-time correlations of the turbulence flow. In 2006 the author and his colleagues began using time-resolved PIV to obtain velocity spectra, pushing the sophistication of the statistics that are available for code validation. The jet noise produced by these flows have been measured in the same facility as the flow measurements, and some near-field acoustic array measurements have also been made to add to the knowledge base.

Besides reviewing the data available to the LES community, this paper will suggest ways to evaluate the datasets being produced by PIV. The PIV technique, while having huge advantages over other flow diagnostic techniques, is not without its weaknesses. These will be covered, based on personal experience, to allow numericists to better understand the uncertainties in the data and properly evaluate the accuracy of their codes. The point of the paper is to help with the dialog between numericists and experimentalists so that both understand the strengths and weakness of their respective tools and needs.

\section{What experimental data are available for validation?}

For the past decade or so the NASA Aeronautics Research Mission Directorate has been funding the development of flow and acoustic databases for validation of numerical prediction methods. Although extensive databases of jet noise existed before this campaign, there have been many questions raised concerning their quality [3], an issue that has been worked very diligently in the NASA experiments [4]. A systematic set of data allows for more consistent noise modeling and better check of trends against other datasets [5,6]. More importantly, no database of flow measurements existed which covered more than a few flow conditions, few data sets contained measurements more advanced than mean velocities and possibly turbulence intensities, and fewer yet were acquired in the same rig as the jet noise measurements. At the same time as these tests were being done, great strides were being made in applying PIV to large, high speed jets. While it is still faster (and hence cheaper) to acquire noise data, it was possible to acquire PIV over a large number of flow conditions and nozzle geometries. In addition, conventional PIV allows measurement of spatial correlations (at zero time delay) with ease, and with the advent of special techniques, time-resolved PIV has allowed direct measurements of the space-time correlations with sufficient temporal resolution for jet noise work.

In order of hierarchy, the following is a list of quantities of interest that are available through PIV. First, some notation conventions:

$$
\begin{aligned}
& \text { time-average: } \bullet^{-}=\frac{1}{T} \int_{0}^{T} \bullet(t) d t \text {, unsteady component: } \bullet^{\prime}(t)=\bullet(t)-\bar{\bullet} \\
& \text { Mean velocity: } \quad \overline{u_{i}}(\vec{x})=\frac{1}{T} \int_{0}^{T} u_{i}(\vec{x}, t) d t \\
& \text { Variance of velocity: } \quad u_{i}^{\prime 2}(\vec{x})=\frac{1}{T} \int_{0}^{T}\left[u_{i}^{\prime}(\vec{x}, t)\right]^{2} d t \\
& \text { Space-time correlations of velocity: } \quad R_{i j}(\vec{x} ; \vec{\eta}, \tau)=\frac{1}{T} \int_{0}^{T} u_{i}^{\prime}(\vec{x}, t) u_{j}^{\prime}(\vec{x}+\vec{\eta}, t+\tau) d t
\end{aligned}
$$


Lengthscales: $L_{i j}(\vec{x})=\frac{1}{2} \int_{-\infty}^{\infty} R_{i i}\left(\vec{x} ; \eta_{j}, \tau\right) d \eta_{j}$

Convective timescale: $T_{i}(\vec{x})=\frac{1}{2} \int_{-\infty}^{\infty} R_{i i}\left(\vec{x} ; \eta_{\text {peak }}, \tau\right) d \tau$

Space-time correlations of Reynolds stress:

$R_{i j \mu v}(\vec{x} ; \vec{\eta}, \tau)=\frac{1}{T} \int_{0}^{T}\left[u_{i}^{\prime} u_{j}^{\prime}-\overline{u_{i} u_{j}}\right](\vec{x}, t)\left[u_{\mu}^{\prime} u_{v}^{\prime}-\overline{u_{\mu} u_{v}}\right](\vec{x}+\vec{\eta}, t+\tau) d t$

The value of mean and variance of velocity are easily recognized. Space-time correlations of velocity, especially ones limited to only time or only space, have been reported in the past, and certainly its autocorrelation, the unsteady Reynolds stress, is understood by the numerical community. Lengthscales are useful in their own right, namely to those pursuing turbulence model development. Convective timescales are somewhat difficult to obtain, requiring highly resolved data in both space and time in order to track the peak correlation as it decays in space and time. The space-time correlations of Reynolds stress, a bit complicated in its definition, is the kinematic portion of the source term in most acoustic analogies. This fourth order correlation is not directly related to the conventional space-time correlation of velocity, and requires careful workup of the variances to produce, but may be the most important measure for free-space aeroacoustic applications.

Recall that for hot jets the space-time correlations involving velocity are only one of the important source terms prescribed by aeroacoustic theory. The two other terms of interest are correlations of turbulent enthalpy, and its correlation with velocity. Important advances in unsteady temperature measurement have been occurring [7], if not quite at the scale and pace of PIV development. Using Rayleigh scattering spectroscopy, fluctuations in density, temperature, and velocity have been measured simultaneously at a point with high enough sample rate to satisfy acoustic requirements. The measurements discussed in [8] include not only those of temperature autocorrelations, but also velocity autocorrelations and velocity-temperature cross-correlations for a number of hot jets already documented by PIV measurements. Most recently, Raman-based scattering techniques have been employed to measure temperature spectrographically. This technique was recently used to measure temperature at two locations in the jet with different time-delays to obtain space-time correlations of turbulence temperature fluctuations, albeit on a rather sparse grid of spatial and temporal separations.

The following is a list of datasets reported by the NASA Glenn research staff for use in validating CFD codes. There has been a significant outpouring of data since the widespread adoption of PIV in the last ten years from labs across the globe. This list is by no means inclusive and is intended only to orient numericists as to the types of data available. In the reports referenced below representative samples of the data are given-more is available.

\section{A. Round subsonic jet flows}

Reference 18 describes a collection of datasets acquired over many years on the same round single-stream jets with various PIV instrumentation systems. A common matrix of seven flows on lines of constant velocity $(0.5,0.9$, 1.33 relative to ambient speed of sound) and static temperature ratio (cold, 1.76, 2.7) was measured each time a PIV test campaign was run in the small hot jet rig (SHJAR) at NASA Glenn. The paper concentrates on measurements of mean and variance of velocity. Estimates for the uncertainty of the data is obtained by comparing NASA datasets acquired with conventional PIV over many years against a wide array of historical data. Basic jet scaling is used to collapse the data, providing a general fit that can be applied even to flow conditions not directly measured. Many details about how the data were acquired are contained in this paper.

Moving to measurements of higher order statistics, reference 9 discusses the same flow matrix as reference 18. This paper describes the use of a dual PIV system with variable time delays to map out the space-time correlation of velocity in heated jet flows. The sparse number of time delays (namely 7) gave coarse temporal resolution, but did provide the first true space-time correlations by PIV as well as estimates for convection velocity, lengthscales and timescales. Reference 10 used this data to demonstrate the relative temperature independence of the space-time correlation once certain normalizing parameters are accounted for. This data was later surpassed by data obtained using time-resolved PIV, as documented in reference 19. Using time-resolved PIV temporal resolution was increased dramatically as the velocity vector fields were sampled at $10 \mathrm{kHz}$ and a few at $25 \mathrm{kHz}$.

Adding to the database for the matrix of hot subsonic jets, reference 11 describes the Rayleigh scattering technique used to obtain single-point statistics of temperature autocorrelation, velocity autocorrelation, and temperature-velocity cross-correlation in the same matrix of flows as listed above. Favorable comparisons are made between the turbulent velocity statistics measured using Rayleigh scattering and using PIV, lending credence to the measurements of velocity-temperature correlation. These measurements of turbulent temperature remain unmatched, 
but are being duplicated by current tests using a Raman scattering instrumentation system operating in the vibrational mode. These ongoing experiments will add a second spatial measurement point and time delays to produce a sparse space-time correlation of temperature fluctuations.

Far-field acoustic spectra of the flows documented in the above references are presented in reference 4.

\section{B. Round supersonic jet flows}

Moving to flows with shocks, reference 12 describes tests of convergent and ideally expanded convergentdivergent nozzles with single-stream supersonic flows. A sparse matrix of six heated flows on lines of constant Mach number $(1.05,1.185,1.4)$ and constant velocity $(1.18,1.4,1.8$ relative to ambient speed of sound) was documented. This paper used time-resolved PIV to present not only mean and turbulent velocities, but also velocity spectra from shock-containing and shock-free jets. The paper contains analysis that shows how mean and turbulent flow fields of shock-free jets can be collapsed. Minimal far-field acoustic spectra are presented in the paper but do exist for all flows measured with PIV.

Conventional PIV measurements of supersonic dual-stream jets have also been taken, although they have not yet been presented in the open literature. The test matrix consists of convergent and ideally expanded nozzles with core flows having the same conditions as explored in reference 12 but with an annular mixer separating a cold bypass stream with bypass ratios of $0.2,1.0$, and 2.0. The far-field acoustic spectra for these cases have been presented in reference 13 in which the influence of the bypass flow on broadband shock noise was studied.

\section{Non-axisymmetric subsonic jet flows}

In 2002 there was an interest in validating Reynolds-Averaged Navier-Stokes CFD for chevron nozzles. Reference 14 describes PIV measurements made on simple chevron nozzles of various geometric parameters. The tests used the same single-stream, subsonic heated flows as reference 18. The paper describes the early application of stereo PIV in a cross-stream orientation and documents mean and turbulent velocity fields along with mean axial vorticity in the chevron nozzle flows. Corresponding far-field acoustic data is described in reference 15.

\section{Non-axisymmetric supersonic jet flows}

The data described in reference 16 had direct applicability to the unique problem of under-exanded jet flows from military convergent-divergent nozzles. Five chevron designs (from a parametric matrix of 10 designs) were applied to a model of a military, dual-stream nozzle. Several underexpanded flow conditions near those of interest to tactical aircraft at takeoff were acquired. PIV data was acquired with both streamwise and cross-stream stereo PIV and the paper presents mean and turbulent velocities and mean axial vorticity. Far-field acoustic data for the entire parametric matrix is presented in reference 17.

This list documents the systematic acquisition of a comprehensive flow and acoustic database for jet flows, suitable for code validation and aeroacoustic model development. The database is being added to, expanding primarily in geometrical complexity with the addition of a family of rectangular nozzles planned for the near future. There are also datasets involving PIV measurements of a mixer-ejector and fluidic slot injection being analyzed for future reporting.

\section{How good are the datasets?}

\section{A. Experimental Cross-Validation}

Collaboration is required between experimentalists and numericists to assure that accuracy of data is properly addressed. Ideally, validation requires that uncertainties in experiments and uncertainties in simulations are quantified, and for the data-simulation enterprise to be successful the two must agree within their respective uncertainty margins. Realistically, neither cutting edge experiments and nor cutting edge simulations are currently capable of the magnitude of effort required to both achieve a measurement/solution and quantify its uncertainty. Thus the need for communication of the qualitative measures of uncertainty at this time.

In experiments, some uncertainty can be assessed by cross-facility comparisons and by repeatability. Clearly, experiments that cannot be repeated in the same facility and then in other facilities are not trustworthy. So users of experimental data have to cross-compare results as a first step to establishing credibility of the dataset being used to validate their simulations. Just finding a measurement that best matches the simulation and only comparing with that data is not sufficient!

As an example of validating PIV by repeatability, consider Figure 1 which shows profiles of mean and turbulent velocity measured in the same jet rig over the course of seven years in seven different experiments, each employing different instrumentation as PIV technology progressed. This work was documented in [18]. An attempt was made, 
assuming the variability to have been caused by independent sources of error arising from the various measurement systems, to establish uncertainty bands about the various profiles. This type of analysis is not possible for every measurement, but should be pursued by every experimentalist for a limited number of flows to establish a baseline and expected repeatability for their facility.
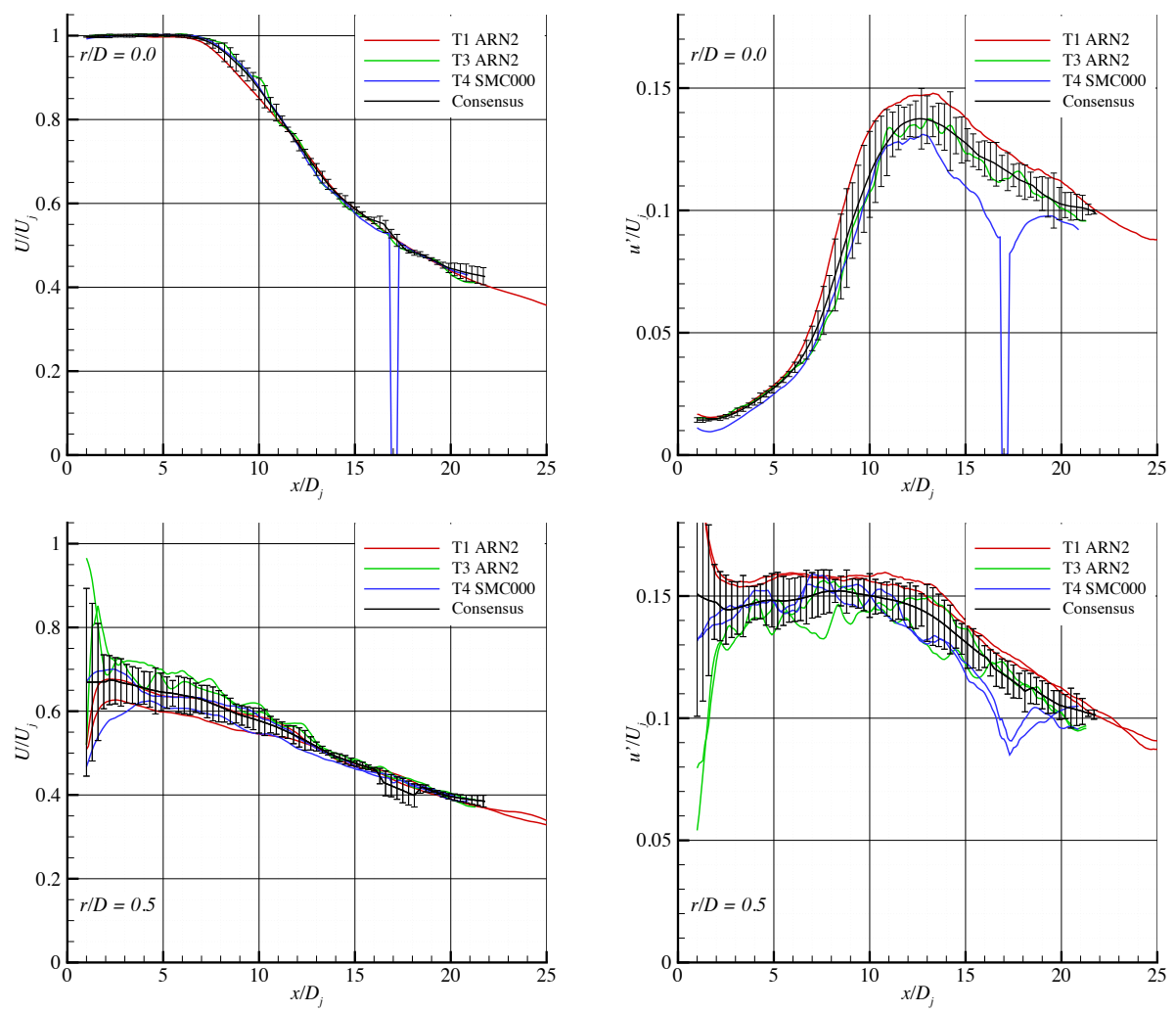

Figure 1 Axial profiles, axial mean and turbulent velocities on the centerline (top) and on the lipline (bottom). $\mathbf{U}_{\mathbf{j}} / \mathbf{c}_{\infty}=\mathbf{0 . 9}$, cold.

For experimentalists it is also important that new measurement systems be cross-validated against older, established measurements. Three examples are given here, a comparison of PIV against hotwire and laser Doppler velocimetry (LDV) data for turbulence intensity and for space-time correlations in a low speed cold jet, and a comparison of shock structures obtained with PIV and shadowgraphy. First, consider Figure 2, extracted from reference 18, where several PIV datasets acquired at NASA Glenn are compared against historical published data acquired using hotwire and LDV. The PIV data mostly sits comfortably within the scatter of the earlier measurements. One important note is that these data have been normalized in the axial location by their potential core lengths $\mathrm{X}_{\mathrm{C}}$ so as to provide a better collapse over the range of Mach numbers included. To quantify the comparison, the disparity in the peak turbulence, both along the centerline at $\mathrm{x} / \mathrm{X}_{\mathrm{C}}=1.5$ and at peak gradient location in the radial profile plot is roughly $\pm 0.1 \%$ out of $15 \%$. 

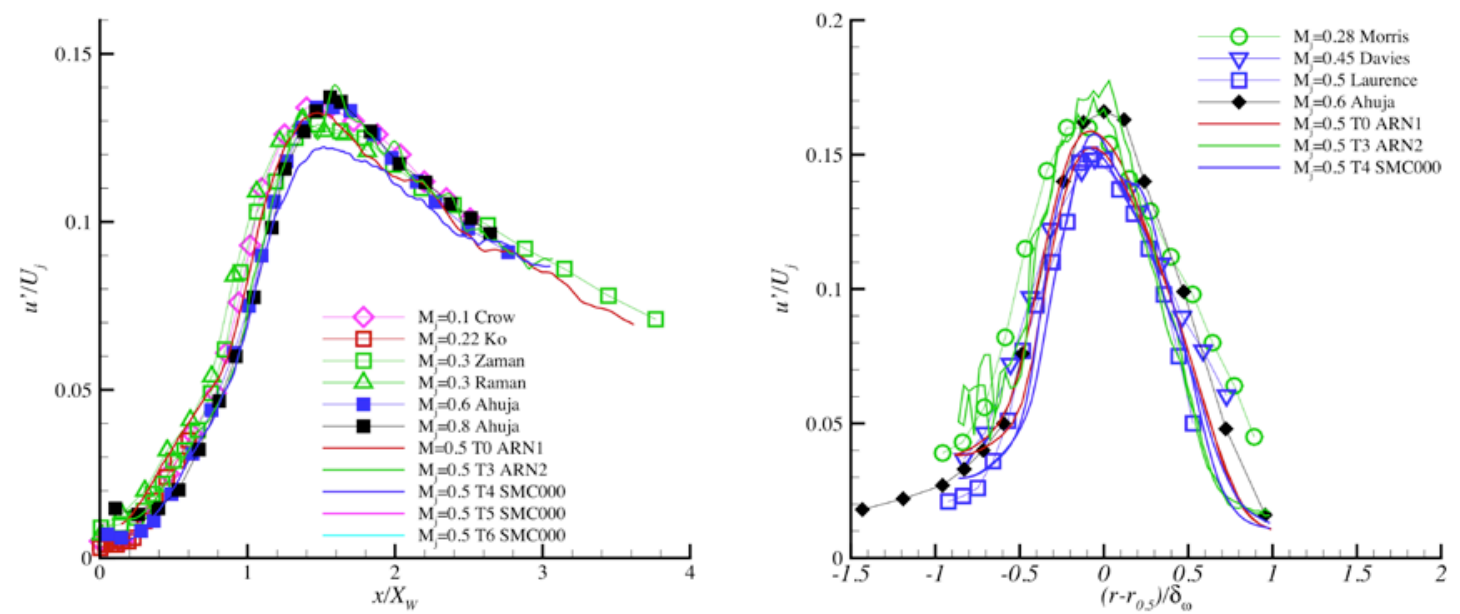

Figure 2 Plots of axial turbulent velocity (rms), in axial profile along centerline (left) and in radial profile at $x / D=4$ (right); low subsonic cold jet: (setpoint 3 ) $U_{j} / c_{\infty}=0.5$, unheated.

Next, consider a limited selection of space-time correlations of velocity from time-resolved PIV data [19] compared with hotwire data of Morris and Zaman [20]shown in Figure 3. The figure shows how the temporal correlation of axial component $R_{11}$ of velocity changes with different spatial separations in a cold, low Mach number jet. The two figures are similar, which is reassuring, but in fact close examination shows that the hotwire correlations are not as large in magnitude as those of the PIV measurements. Note that the hotwire data does not contain a curve for zero separation, but has a progression of separations whose peak correlations linearly decay with separation. The PIV data has the same behavior, excepting that the PIV data was not sampled at as high a rate in time and hence sometimes misses sampling the peak correlation for a given separation. By extrapolating the hotwire data to the zero separation correlation peak one sees that it falls well shy of the proper value of 1 . Thus it seems that the PIV data, while exhibiting the same character as the hotwire data, is actually more accurate. This example was chosen intentionally because it shows one of the strengths of PIV - the ability to smoothly vary spatial measurement location without probe interference - and one of its weaknesses - the discrete nature of the measurement. It also shows the sort of analysis that needs to be used in evaluating experimental data.
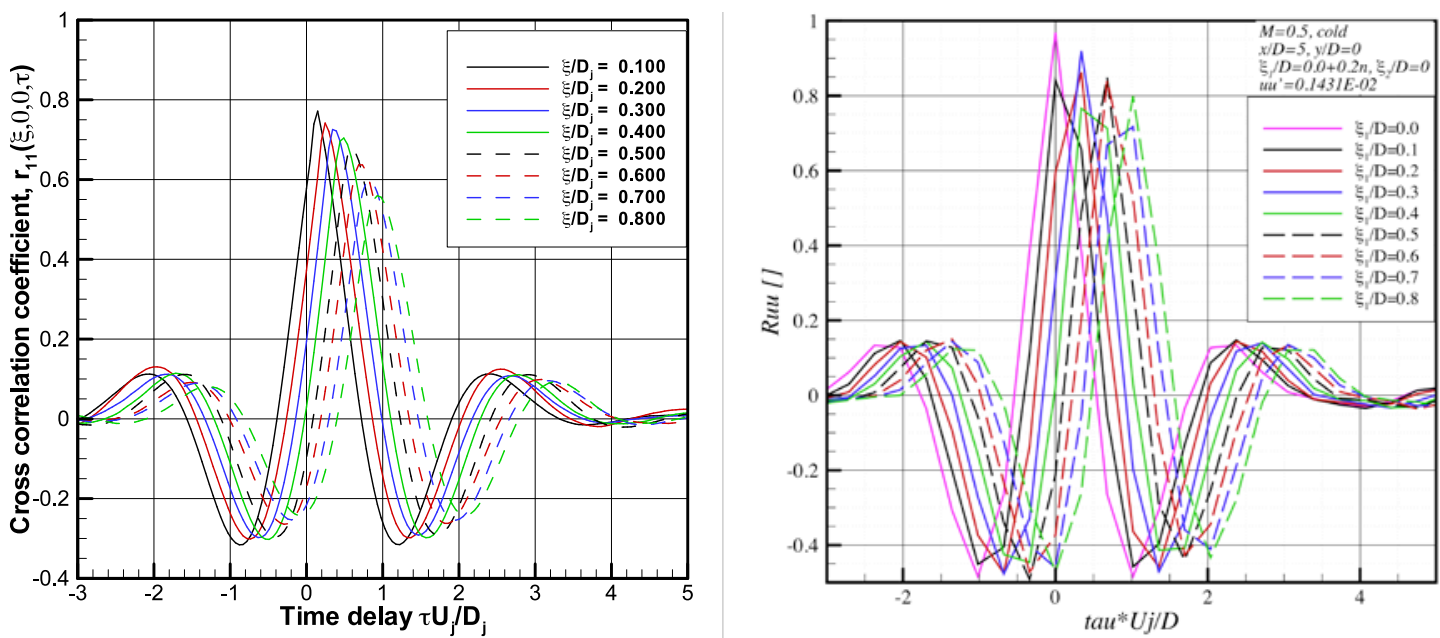

Figure 3 Comparison of Morris \& Zaman data (left) with time-resolved PIV data (right): $R_{11}$ at x/D = 5, y/D = 0 with radial separation $\xi_{\mathrm{y}} / \mathrm{D}=\mathbf{0}$.

Another example where PIV data were validated against more conventional instrumentation is the comparison of shock structure measured with shadowgraphy. While it would seem that capturing the mean velocity field would be much easier than high-order turbulence statistics, this comparison addresses the potential error of particle lag and optical distortion, two commonly suspected sources of error in PIV measurements. However, based on analysis of drag on spheres, such problems are not severe as long as reasonable seeding is used. However, it is reassuring in 
Figure 4 to see the degree to which the occlusions from the refraction of the shock cells line up with the deceleration fronts in the PIV data.

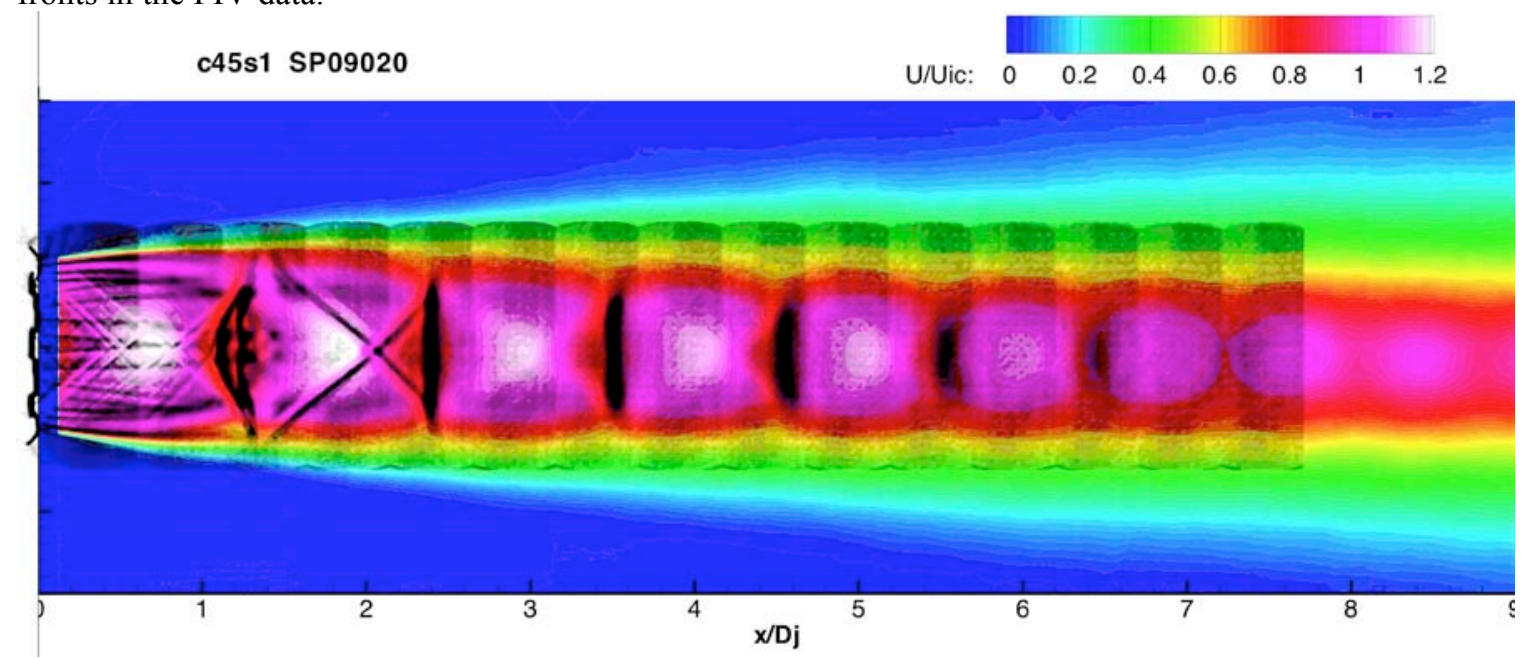

Figure 4 Contour plots of mean axial velocity from PIV measurements (color contours) overlaid with shadowgraph images (black). Flow is from tabbed convergent nozzle operating at $M=1.4$, isothermal flow conditions.

\section{B. Interpreting PIV data}

But how to judge data accuracy among different sources, or if only one data source exists, or the measurement is not one that can be obtained by other measurement systems? In PIV measurements, the image processing software can deliver statistics that attest to the reliability of individual measurement locations. These need to be reported by experimentalists more often to help make these determinations. Just as numericists have their various filters and internal modeling which make some codes more robust or more accurate, so it is with experimentalists. Since most PIV processing suites employ a variety of schemes to enhance the basic particle image correlation that is the basis for the measurement, not all experimentalists will arrive at the same number even if they start with the same images. And analogous to the sensitivity of CFD to grid generation, the seeding, lighting, and camera quality, which collectively contribute to the quality of the images, are the most crucial aspects of PIV experimentation and the biggest determinant of data accuracy. These effects are hard to quantify in the "Facilities and Instrumentation" sections of papers and reports, just as it is difficult to judge the accuracy of a numerical solution by the image of the grid printed in a journal paper. However, a few internal statistics are available from PIV processing which can be used to evaluate whether local features of the flow are real or artifacts.

For instance, with stereo PIV two 2-D projections of the vector displacement field from the two camera views are acquired. These are input into a three-dimensional warping to map the four vector components at a point in the laser plane to a 3-D vector. The residual in this fit, essentially a measure of how well the two common vector components agree, is an internal check on the accuracy of the image processing step. This residual is reported in most PIV software. This is done for every point in every time instance measured. When these data are processed into statistics this residual can be checked before including the point in the statistic, removing points from the averaging used in the statistic. In regions where image quality is poor a significant number of points acquired are left out of the statistic, an indicator that even the remaining points are likely tainted. By mapping out the fraction of instantaneous vectors that were included in the statistic, local blemishes in the data can be flagged. If an interesting feature corresponds to a region where the fraction of valid vectors was low, then this feature would be highly suspect.

An example of using the fraction of accepted instantaneous vectors in the statistic is given in Figure 5. On the left is a plot of axial turbulence intensity $\mathrm{u}^{\prime} / \mathrm{U}$ measured along the centerline of a $\mathrm{M}=0.9$ cold jet in three different experiments. Two of the datasets agree up to $\mathrm{x} / \mathrm{D}=12$ or so, but one exhibits a strong dip around $\mathrm{x} / \mathrm{D}=18$. The third measurement is higher than the other two. Which to believe? The plot on the right shows the fraction of accepted points in the average used to create the figure on the left. The dataset with the strong dip at $x / D=18$ show a corresponding precipitous drop in data quality at this same region, casting doubt on the validity of that data in that region. If only the blue curve was available and the CFD did not demonstrate a comparable dip in turbulence, the measure of quality would give good reason to reject it in the comparison with CFD. Or, if multiple datasets were 
available, the quality metric could be used as a weighting factor in averaging the datasets, reducing the impact of the bad data without throwing out the entire dataset.
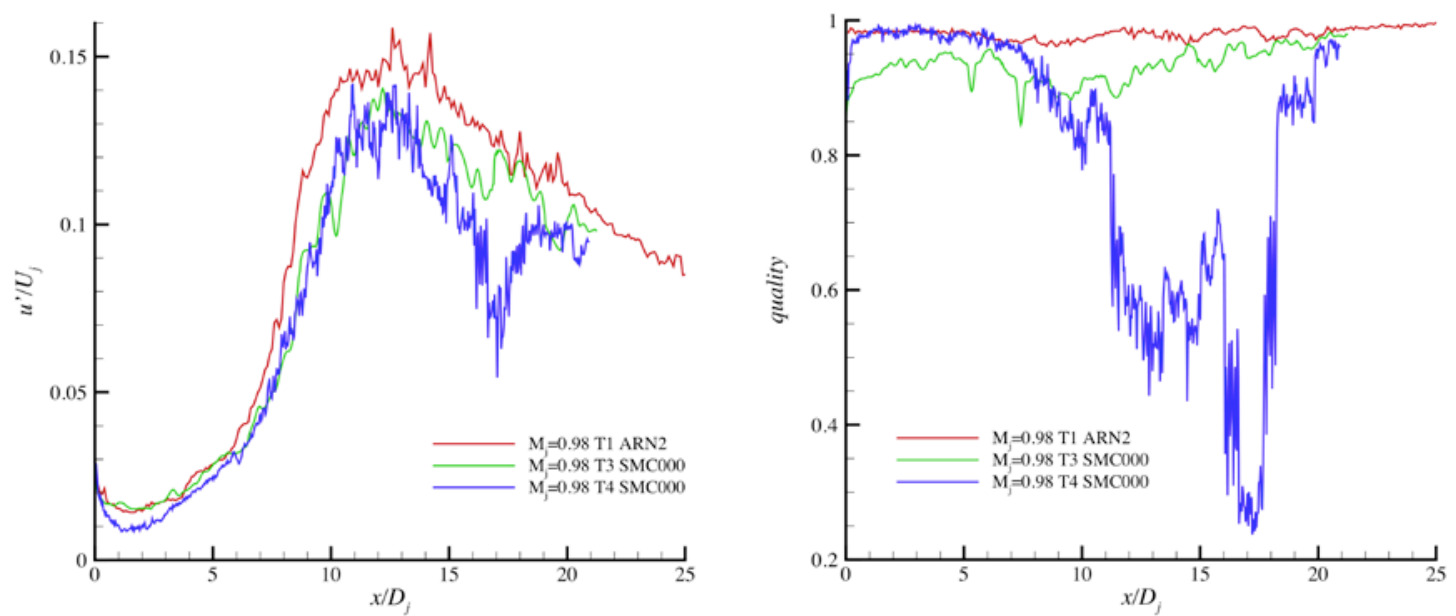

Figure 5 Example of using 'quality' metric to confirm bad data within a dataset.

Other aspects of PIV image processing need care when using advanced processing algorithms that allow increased productivity from measurements in poor quality images. Ideally, every measurement of particle displacement in a subregion is made independent of other subregions and of other measurements at the same location at other times. However, most image processing schemes start with assessment of average displacement over a larger subregion than the final resolution and using this result to refine the search for correlation peaks in subsequent passes over the image. In some advanced processing schemes nearby subregions are consulted if the image displacement correlation is suspiciously low in a given subregion. If nearby subregions are different, the image displacement correlation is investigated to see if a local maxima is found at the same displacement as the neighbor. This is especially useful when seed quality is spotty or if some regions have fixed background images. The only way to determine if this practice is reducing statistical error by increasing the number of valid samples or producing false results is to process the data several ways to see how sensitive the answer is to the thresholds inherent in the processing. Think of it as being analogous to grid refinement studies in CFD, a practice that is required before the simulation is considered valid.

The point of describing these aspects of PIV processing for non-experimentalists is so that they know what questions to ask of the providers of the data. Just as most people who use CFD results know enough to ask about convergence criteria, grid refinement studies, and other details before blindly accepting a CFD solution, numericists need to ask about details of the processing of the PIV they use in validation.

\section{Summary}

Large Eddy Simulations hold the promise of delivering everything aeroacousticians have asked for in their quest to manage flow noise, but the promise is contingent upon the accuracy of LES being validated and its limitations established. Given the high demands placed on LES codes by the needs of aeroacoustics, more accuracy and a higher range of scales is required than is the case in other applications of LES. Validation must therefore be done on higher order statistics of the computed flow. The paper advocates for a layered set of validations being performed on LES solutions to ascertain the limitations of codes, as well as standardized internal checks to measure the accuracy of each solution. Several experimental datasets exist for various statistics of interest to aeroacoustics, and for varying degrees of geometric complexity. Key to choosing the dataset is the ability to cross-check the data and evaluate whether features are artifacts of the experiment or interesting aspects of fluid mechanics. Examples of such cross-validation are given, along with some aspects of the internal workings of PIV analysis that allow assessment of data quality. Deliberate and conscientious collaboration between experimentalist and numericist is required so that each can understand the needs and limits of each other. In this way the limitations of LES codes and solutions can be established and the promise of LES be realized.

\section{Acknowledgements}

The author acknowledges the amazing work of his colleagues and the coauthors of the papers referenced herein. The tests described in this report were supported by the several programs under NASA's Aeronautics Directorate: 
the Quiet Aircraft Technology Program, and the Subsonic Fixed Wing and Supersonics Projects of NASA's Fundamental Aeronautics Program. Finally, the author thanks Ed Envia for his thoughtful input to the manuscript.

\section{References}

1 Goldstein, M.E., and Leib, S.J. "The aeroacoustics of slowly diverging supersonic jets," Journal of Fluid Mechanics 600, 291-337 (2008).

2 Khavaran, A. and Kenzakowski, D.C., "Noise Prediction in Hot Jets," AIAA 2007-3640 (2007).

3 Viswanathan, K. "Jet Aeroacoustic Testing: Issues and Implications," AIAA Journal 41, p1674-1689 (2003).

4 Bridges, J. \& Brown, C.A., "Validation of the Small Hot Jet Acoustic Rig for Jet Noise Research," AIAA2005-2846 (2005).

5 Khavaran, A., and Bridges, J., "SHJAR jet noise data and power spectral laws," NASA TM-2009-215608 (2009).

6 Khavaran, A. and Bridges, J., "Jet Noise Scaling in Dual Stream Nozzles," AIAA-2010-3968 (2010).

7 Panda, J. \& Seasholtz, R. G. "Experimental investigation of density fluctuations in high-speed jets and correlation with generated noise," Journal of Fluid Mechanics 450, pp. 97-130 (2002).

8 Mielke, A.F., Elam, K.A., "Dynamic Measurement of Temperature, Velocity, and Density in Hot Jets Using Rayleigh Scattering," Experiments in Fluids 47, pp. 673-688 (2009)

9 Bridges, J. \& Wernet, M.P. "Measurements of the aeroacoustic sound source in hot jets," AIAA 2003-3130 (2003).

10 Bridges, J., "Effect of Heat on Space-Time Correlations in Jets," AIAA-2006-2534 (2006).

11 Mielke, A.F., Elam, K.A., Sung, C.J., "Multiproperty Measurements at High Sampling Rates Using Rayleigh Scattering," AIAA Journal 47, pp. 850-862 (2009).

12 Bridges, J. and Wernet, M.P., "Turbulence Associated with Broadband Shock Noise in Hot Jets," AIAA2008-2834 (2008).

13 Bridges, J., "Broadband Shock Noise in Internally-Mixed Dual-Stream Jets," AIAA-2009-3210 (2009).

14 Opalski, A.B., Wernet, M.P., \& Bridges, J., "Chevron nozzle performance characterization using stereoscopic DPIV," AIAA-2005-0444, (2005). (2004)

15 Bridges, J. and Brown, C.A., "Parametric testing of chevrons on single flow hot jets," AIAA-2004-2824

16 Bridges, J. and Wernet, M.P., "PIV measurements of chevrons on F400-series tactical aircraft nozzle model," AIAA-2011-XXXX (presented at the 2011 AIAA ASM, Orlando, 2011).

17 Henderson, B., and Bridges, J. (2010). "An MDOE Investigation of Chevrons for Supersonic Jet Noise Reduction," AIAA 2010-3926 (2010).

18 Bridges, J. and Wernet, M.P., "Establishing Consensus Turbulence Statistics for Hot Subsonic Jets," AIAA2010-3751 (2010).

19 Bridges, J. and Wernet, M.P., "Effect of Temperature on Jet Velocity Spectra," AIAA-2007-3628 (2007).

20 Morris, P.J. and Zaman, K.B.M.Q., "Velocity Measurements in Jets with Application to Noise Source Modeling," AIAA-2009-17 (2009). 